\title{
Modeling the Step-like Response in the Upper Limbs of Hemiplegic Subjects for Evaluation of Spasticity
}

\author{
Takanori Uchiyama* Member \\ Ryusei Uchida ${ }^{* *} \quad$ Non-member
}

\begin{abstract}
The purpose of this study is to develop a new modeling technique for quantitative evaluation of spasticity in the upper limbs of hemiplegic patients. Each subject lay on a bed, and his forearm was supported with a jig to measure the elbow joint angle. The subject was instructed to relax and not to resist the step-like load which was applied to extend the elbow joint. The elbow joint angle and electromyogram (EMG) of the biceps muscle, triceps muscle and brachioradialis muscle were measured. First, the step-like response was approximated with a proposed mathematical model based on musculoskeletal and physiological characteristics by the least square method. The proposed model involved an elastic component depending on both muscle activities and elbow joint angle. The responses were approximated well with the proposed model. Next, the torque generated by the elastic component was estimated. The normalized elastic torque was approximated with a dumped sinusoid by the least square method. The reciprocal of the time constant and the natural frequency of the normalized elastic torque were calculated and they varied depending on the grades of the modified Ashworth scale of the subjects. It was suggested that the proposed modeling technique would provide a good quantitative index of spasticity as shown in the relationship between the reciprocal of the time constant and the natural frequency.
\end{abstract}

Keywords: elasticity, viscosity, elbow, spasticity

\section{Introduction}

Spasticity is a typical symptom after cerebral stroke. The grade of spasticity is often evaluated by the modified Ashworth scale (AS) because the measurement is simple, and no special equipment is needed. The grade is based on the relationship between range of motion and resistance force during extension/flexion of the joint. However, it is a qualitative index and sometimes fluctuates depending on a doctor's experience. Measuring the resistance force during extension/flexion corresponds to evaluating the visco-elastic property of the joint. Many reports have evaluated the visco-elastic property of joints ${ }^{(1)-(7)}$. These studies have evaluated the resistant force ${ }^{(1)}$, the resonance frequency ${ }^{(2)}$, the dynamic stiffness transfer function ${ }^{(3)}$, the Nyquist diagram of stiffness ${ }^{(4)(5)}$, the EMG ${ }^{(6)}$ and parameters related to stretch reflex ${ }^{(7)}$. All of them used the torque motor or kinematic equipment in order to apply sinusoidal oscillation or constant velocity extension to the joint. The torque motor or kinematic equipment provide an ideal input to the system (spastic limbs) for evaluating the system characteristics however these methods have not been widely used because of lack of available equipments.

\footnotetext{
* Keio University

3-14-1, Hiyoshi, Kohoku-ku, Yokohama 223-8522, Japan

** Kanto-Rosai Hospital

1-1, Sumiyoshicho, Kizuki, Nakahara-ku, Kawasaki 211-8510, Japan
}

In this study, we did not use kinematic equipment. Instead, we measured the step-like response of the elbow joint caused by an external load which was the gravitational force of a weight. The step response is one of the effective input to evaluate the system. In this study, the response was not a strict step response because the weight had non-zero acceleration. Therefore we call the response "step-like response." The purpose of this study was to develop a modeling technique of the step-like response for quantitatively evaluating spasticity in the upper limbs of hemiplegic patients.

\section{Method}

2.1 Measurement The step-like response of elbow extension caused by an external load was measured. A schematic diagram of the experimental setup is shown in Fig. 1. The measurements were performed with two healthy subjects (A and B) and twelve hemiplegic subjects $(\mathrm{C}-\mathrm{N})$. All gave informed consent. The ages and the modified Ashworth scale grades of the subjects are shown in Table 1.

Each subject lay on a bed, his (her) forearm supported with a jig to measure the elbow angle. The gravitational force of his (her) arm and the jig were compensated by a balance weight. The weight of the subject's arm and hand was calculated from his (her) weight, and the length of his (her) arms and hands ${ }^{(8)}{ }^{(9)}$. The subject could flex and extend his (her) elbow joint in the vertical plane. The subject was instructed to relax and not to resist the step-like external load which was applied 


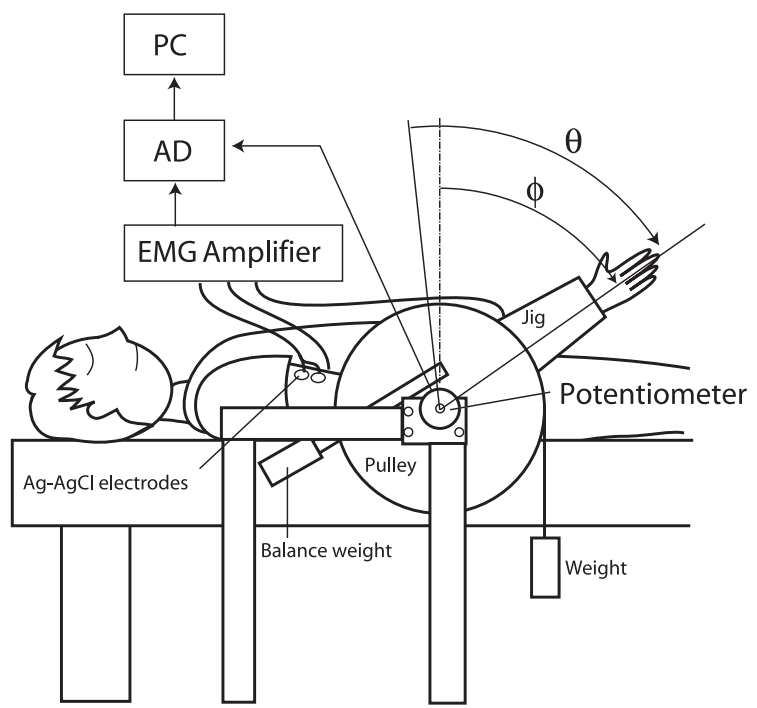

Fig. 1. Schematic illustration of setup.

Table 1. Subjects, modified Ashworth scale and weight.

\begin{tabular}{c|c|c|c} 
Subject & Age & AS & weight $(\mathrm{g})$ \\
\hline A & 46 & - & 400,500 \\
B & 38 & - & $500,600,700$ \\
C & 45 & 3 & $1500,1700,1900$ \\
D & 71 & 1 & $40^{1,2}, 60^{1,2}$ \\
E & 59 & 2 & $300^{1,2}, 400^{1,2}, 500^{1,2}, 700^{2}$ \\
F & 61 & 3 & $600^{1,2}, 800^{1,2}, 1000^{2}$ \\
G & 56 & 1 & $40^{1,2,3}, 70^{2,3}, 100^{2}$ \\
H & 49 & 2 & $300,500,700$ \\
I & 61 & 2 & $1300,1500,1700$ \\
J & 57 & 0 & 40,70 \\
K & 64 & 4 & 2000,2500 \\
L & 59 & 0 & 200 \\
M & 53 & 0 & 160 \\
N & 72 & 1 & 1500
\end{tabular}

1 the first measurement

2 the second measurement after one month of the first one

3 the third measurement after one month of the second one

to extend his elbow joint. The step-like external load was the gravitational force of a weight hung on a pulley. Weights were shown in Table 1 . These weights allowed observation of a sufficient amplitude of the step-like response without causing full extension. The initial elbow angle was approximately $100^{\circ}$, and the forearm was supported by an experimenter who released the forearm at an arbitrary time.

The elbow angle was measured with a potentiometer attached to the axis of the pulley. EMG was obtained with $\mathrm{Ag}-\mathrm{AgCl}$ surface electrodes. Electrodes $10 \mathrm{~mm}$ in diameter were taped to the skin $15 \mathrm{~mm}$ apart for bipolar recording. EMGs were recorded from the biceps muscle, triceps muscle and brachioradialis muscle. EMG signals were full-wave rectified and smoothed with a secondorder low pass filter $\left(f_{c}=2.6 \mathrm{~Hz}\right)$ to obtain integrated EMG (IEMG). The measurements were repeated at least three times for each weight.

2.2 Musculoskeletal Model There are several musculoskeletal models of the upper limb ${ }^{(10)-(13)}$. Although the details of the models differ slightly, the flexor muscles of the upper limb is regarded as similar to those in the model illustrated in Fig. 2. The elbow joint torque

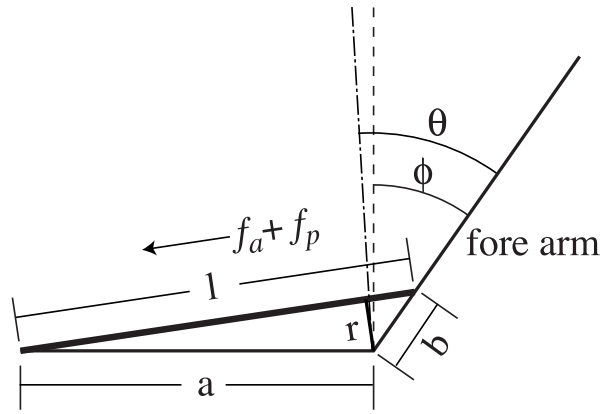

upper arm

Fig. 2. Model of musculoskeletal structure of upper limb.

is equal to the vector product of the force and the moment $\operatorname{arm} r$. The force is generated by activating muscles and elongated passive components such as tendon and connective tissue.

We assumed that the force, $f_{a}$, generated by the elbow flexor muscle at constant activation level was proportional to their stretch length. For simplicity, we assumed that (a) the passive components had a natural length $l_{0}$ when the elbow angle was $90^{\circ}(\phi=0)$ and the passive components began to generate force, $f_{p}$, as the extension of the elbow joint; (b) $f_{p}$ was also proportional to their stretch length, $l-l_{0}$.

The muscle length $l$ and the moment arm $r$ in Fig. 2 can be calculated as Eqs. 1 and 2:

$$
\begin{aligned}
l & =\sqrt{(b \cos \phi)^{2}+(a+b \sin \phi)^{2}} \ldots \ldots \ldots \ldots \ldots \ldots \ldots \ldots \ldots \ldots \ldots \ldots \ldots \ldots \ldots \ldots
\end{aligned}
$$

where $a$ and $b$ are the distances between the axis of joint rotation and origin/insertion of the muscle, respectively.

The torque $T$ generated by the muscle was calculated as follows:

$$
\begin{aligned}
T & =\left(f_{a}+f_{p}\right) r \\
& =c\left(l-\sqrt{a^{2}+b^{2}}\right) \frac{a b \cos \phi}{l}
\end{aligned}
$$

where $c$ is the elastic coefficient of the muscle.

The torque $T$ is approximated by the second-order polynomials of the angle $\phi$ as shown in Eq. 4:

$$
\begin{aligned}
T & =c\left(\frac{a b}{a^{2}+b^{2}} \phi-\frac{3}{2} \frac{a^{2} b^{2}}{\left(a^{2}+b^{2}\right)^{2}} \phi^{2}+\cdots\right) a b\left(1-\frac{1}{2} \phi^{2}+\cdots\right) \\
& \simeq c a b\left(\frac{a b}{a^{2}+b^{2}} \phi-\frac{3}{2} \frac{a^{2} b^{2}}{\left(a^{2}+b^{2}\right)^{2}} \phi^{2}\right) \cdots \cdots \cdots \cdots(4)
\end{aligned}
$$

Although the muscles are activated, the moment arm has a dominant effect on the torque generated by the elbow flexor muscles. We reported that the moment arm vs. elbow angle curve has a similar shape to the torqueangle curve ${ }^{(14)}$.

2.3 Modeling of Step-like Response The equation of motion around the elbow joint is Eq. 5:

$$
I \ddot{\theta}(t)+\eta \dot{\theta}(t)+k \theta(t)=h(t)
$$

where $I$ is the inertia of the forearm and the jig involving the pulley and the balance weight; $\theta(t)$ is the relative elbow angle; $\eta$ is the viscous coefficient and $k$ is the elastic 
coefficient. $h(t)$ is the torque generated by the external load:

$$
\begin{aligned}
h(t) & =m(g-\ddot{x}) R \\
& =m g r-m R^{2} \ddot{\theta} .
\end{aligned}
$$

where $R$ is the radius of the pulley, $m$ is the weight, $g$ is the gravitational constant and $\ddot{x}$ is the acceleration of the weight. The relative elbow angle $\theta(t)$ is zero at the onset of the extension.

Substituting Eq. 6 to Eq. 5, Eq. 7 is derived:

$$
I^{\prime} \ddot{\theta}(t)+\eta \dot{\theta}(t)+k \theta(t)=m g r
$$

where $I^{\prime}\left(=I+m R^{2}\right)$ is the net inertia.

We assumed that the elastic coefficients were proportional to both the elbow angle and the muscle activities (IEMG) as shown in Eq. 8 because it was reported that the elasticity was proportional to the muscle force and the muscle force was proportional to the IEMG ${ }^{(15)(16)}$ :

$$
k=k_{0}+k_{1} \theta(t)+a_{1} e_{1}(t)+a_{2} e_{2}(t)+a_{3} e_{3}(t)
$$

where $e_{1}(t), e_{2}(t)$ and $e_{3}(t)$ are IEMGs of the biceps muscle, triceps muscle and brachioradialis muscle, respectively. The parameter $k_{0}$ is a component of the elastic coefficient without dependency on both elbow angle and the IEMG. The parameter $k_{1}$ is a coefficient of the component of the elastic coefficient proportional to the elbow angle. The parameters $a_{1}, a_{2}$ and $a_{3}$ are coefficients of the components of the elastic coefficient proportional to the IEMGs of biceps muscle, triceps muscle and brachioradialis muscle, respectively.

The net inertia $I^{\prime}$, viscous coefficient $\eta$ and parameters related to elastic coefficient, $k_{0}, k_{1}, a_{1}, a_{2}, a_{3}$, were calculated by the least squares method (Appendix).

The elbow angle was estimated, using these calculated parameters, by the Runge-Kutta method and was compared with the observed elbow angle in order to evaluate performance of the proposed model.

2.4 Normalized Elastic Torque We supposed that resistance force during extension/flexion of the elbow joint of the hemiplegic subject was caused by the changes of the viscoelasticity depending on muscle activities and the elbow angle. The activating muscle has large elastic coefficient, $c$, in Eq. 3. The elastic component was evaluated as follows. First, the elastic torque was calculated by subtracting torques generated by the net inertia and viscosity from the total torque $\mathrm{mgr}$. The elastic torque was normalized by the total torque as shown in Eq. 9:

$$
e(t)=\frac{m g r-I^{\prime} \ddot{\theta}(t)-\eta \dot{\theta}(t)}{m g r}
$$

where $e(t)$ is the normalized elastic torque.

Then the normalized elastic torque was approximated with a dumped sinusoid in Eq. 10:

$$
A \exp (-t / \tau) \sin \left(2 \pi f_{0} t+\psi\right)+1.0
$$

where $A$ is the relative amplitude, $\tau$ is the time constant of dumping, $f_{0}$ is the natural frequency and $\psi$ is the phase. The parameters, $A, \tau, f_{0}$ and $\phi$, were estimated by the least square method using a commercial software based on Levenberg-Marquardt method.

Finally, the time constant and the natural frequency were evaluated.

\section{Results}

3.1 Step-like Response Fig. 3 represents examples of the observed elbow angle and IEMGs of the step-like response. The top left panel (a) gives the elbow angle of Subject B (healthy). The bottom left panel (b) shows the IEMGs of Subject B. The solid line, the dotted line and the broken dotted line denote the IEMGs of biceps brachii muscle, brachioradialis muscle and triceps brachii muscle, respectively. The top right panel (c)
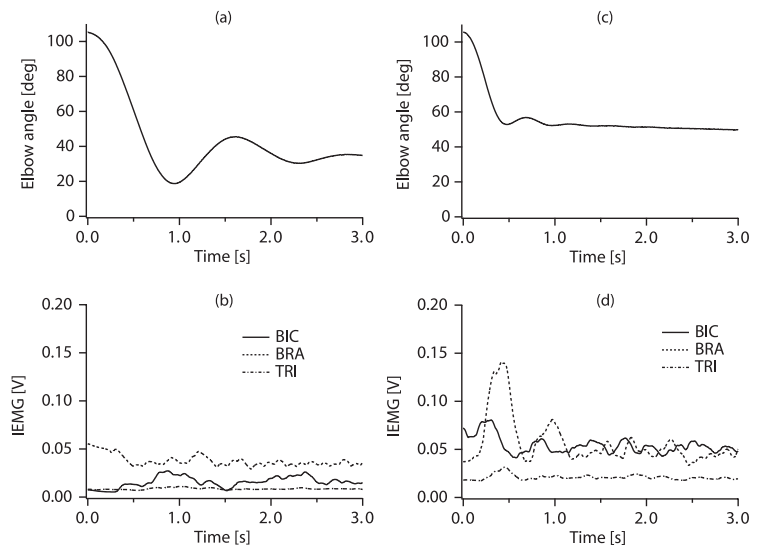

Fig. 3. Time course of elbow angle and IEMG. Top and bottom panels represent elbow angle and IEMG, respectively. (a) and (b) Subject B, 700 g; (c) and (d) Subject C, $1500 \mathrm{~g}$.
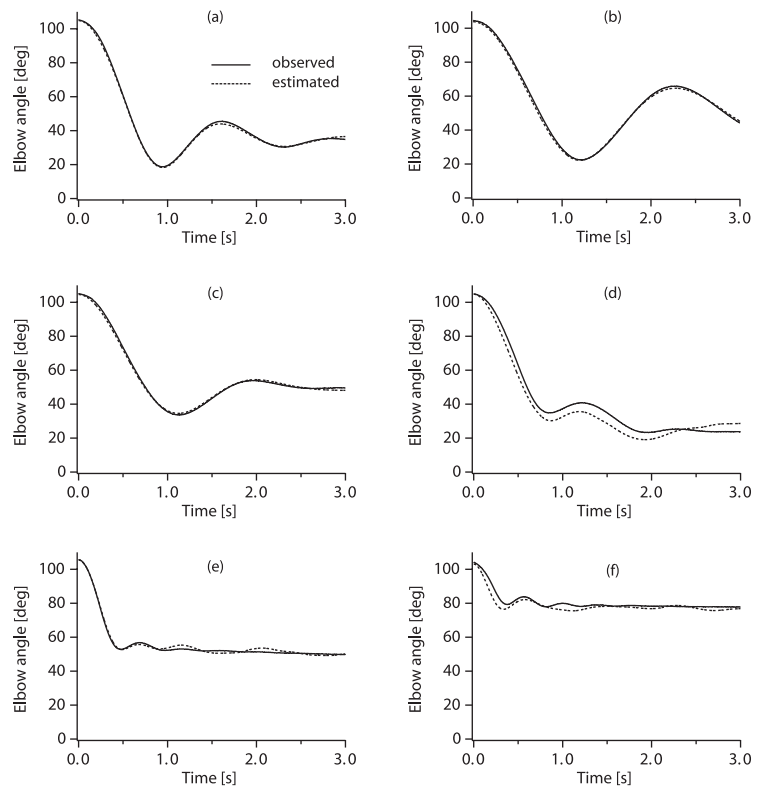

Fig. 4. Examples of step-like response and estimation. The solid lines denote the observed elbow angle. The dotted lines denote the estimated angle. (a) Subject B, $700 \mathrm{~g}$, healthy; (b) Subject L, $200 \mathrm{~g}$, AS 0; (c) Subject G, 40 g, AS 1; (d) Subject E, 300 g, AS 2; (e) Subject C, 1500 g, AS 3; (f) Subject $\mathrm{K}, 2000 \mathrm{~g}, \mathrm{AS} 4$. 

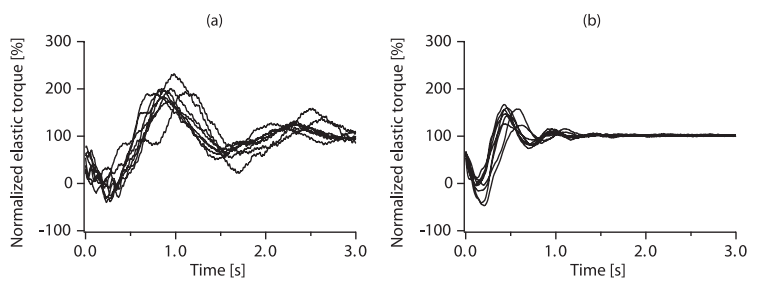

Fig. 5. Normalized elastic torque. (a) Subject B (healthy), (b) Subject C (AS 3).

gives the elbow angle of Subject $\mathrm{C}$ (hemiplegic). In spite of the heavy weight, the extension angle was smaller than that of the healthy subject. The bottom right panel (d) shows the IEMGs of the Subject C. The IEMG of brachioradialis muscle shows a strong peak around $0.4 \mathrm{~s}$ while that of the healthy subject does not.

Fig. 4 shows the typical examples of step-like responses and their approximation by the proposed model. The top left panel (a) shows the response of Subject B (healthy) when the weight was $700 \mathrm{~g}$. The solid line denotes the observed elbow angle. The dotted line denotes the estimated angle. The relative elbow angle was estimated well with the proposed model. The top right panel (b) is the step-like response of Subject L (AS 0) with an applied weight of $200 \mathrm{~g}$. The relative elbow angle was also estimated well. The panels (c), (d), (e) and (f) show the step-like response of Subject G (AS 1), E (AS 2), C (AS 3) and K (AS 4), respectively.

The elbow angle of the hemiplegic subjects declined more rapidly than those of the healthy subjects did. This rapid decline was clear in the subjects whose modified Ashworth scale grades were high (Subject $\mathrm{C}$ and $\mathrm{K}$ ). The oscillating frequency had a tendency to be higher in the hemiplegic subjects of high AS than in the healthy subjects. Theses characteristics suggest an increase in the viscoelasticity of the elbow joint. The elbow angle were estimated well with the proposed model, although different characteristics such as rapid decline and high oscillating frequency were observed.

3.2 Normalized Elastic Torque Fig. 5 represents the normalized elastic torque of Subjects B and C. They had repeatability, although three different weights were applied to the subjects.

Fig. 6 shows typical examples of the normalized elastic torque. Each panel corresponds to the same one in Fig. 4. Solid lines denote the torque calculated from the observed torque. Dotted lines denote the torque approximated with the dumped sinusoid. Table 2 shows the parameters, $A, 1 / \tau, f_{0}$ and $\phi$ of the dotted lines. The normalized elastic torque could be approximated with the dumped sinusoid, although small over/undershoots remained in some cases. For the hemiplegic subjects of high AS, as shown in (e) and (f), the normalized elastic torque declined more rapidly than those of the healthy subjects and hemiplegic subjects of low AS did.

Fig. 7 shows the relationship between the inverse of the time constant and the natural frequency of the normalized elastic torque. The crosses denote the healthy subjects. The open circles denote the subjects whose
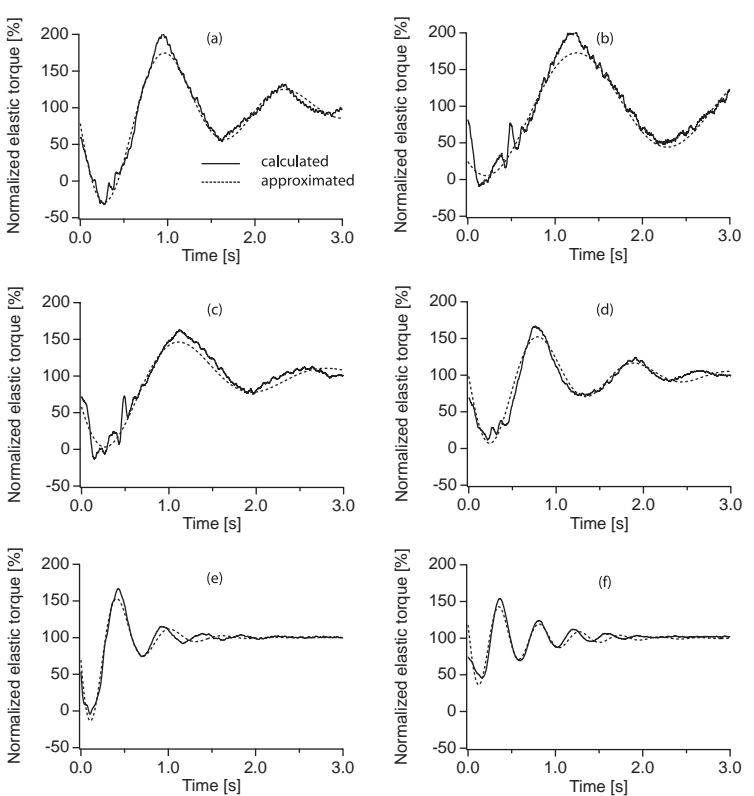

Fig. 6. Normalized elastic torque. Each panel corresponds to the same panel in Fig. 4. Solid lines denote the normalized elastic torque. Dotted lines denote the torque approximated with the dumped sinusoid.

Table 2. The relative amplitude, natural frequency, time constant and phase of the approximated curves shown in Fig. 6

\begin{tabular}{l|rrrr} 
& $A$ & $f_{0}(\mathrm{~Hz})$ & $1 / \tau\left(\mathrm{s}^{-1}\right)$ & $\phi(\mathrm{rad})$ \\
\hline (a) & -1.63 & 0.729 & 0.796 & 0.132 \\
(b) & 1.00 & 0.482 & 0.255 & 3.99 \\
(c) & 1.26 & 0.593 & 0.875 & 9.77 \\
(d) & -1.22 & 0.912 & 1.05 & 0.0137 \\
(e) & -1.54 & 1.66 & 2.52 & 0.200 \\
(f) & -0.796 & 2.17 & 1.73 & -6.51
\end{tabular}

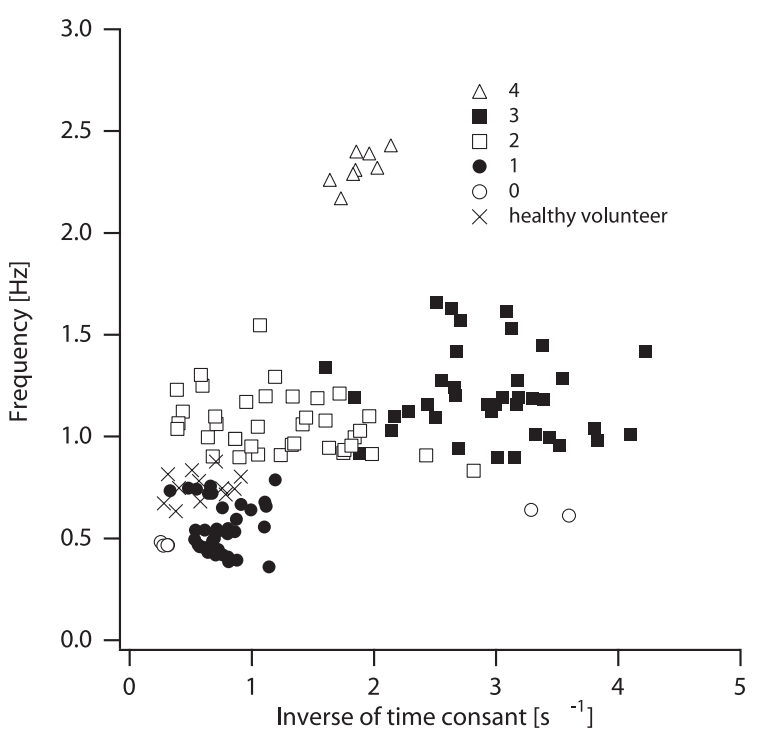

Fig. 7. Relationship between the inverse of the time constant and the natural frequency.

AS was zero. The closed circles, open squares, closed squares and open triangles denote the subjects whose AS were one, two, three and four, respectively.

For high AS subjects, the inverse of the time constant 
was relatively large and the natural frequency was high. For low AS subjects, these values were close to those of the healthy subjects.

\section{Discussion}

4.1 Natural Length and Torque of Passive Components We assumed that the passive components have a natural length at the onset of the elbow extension. The elbow angle at the natural length of the passive components was unknown, although the angle could be the intermediate of extension/flexion. The assumption was not consistent with the explanation of Eqs. $1-4$. However, the assumption did not affect our results greatly.

Fig. 8 shows the relationship among muscle length, moment arm, stretch length, torque and the angle $\phi$. Distances $a$ and $b$ of biceps muscle are $282 \mathrm{~mm}$ and 48 $\mathrm{mm}$, respectively ${ }^{(10)}$. The top left panel (a) shows the relationships between the muscle length and the angle $\phi$. The top right panel (b) shows the relationships between the moment arm and the angle $\phi$. The moment arm reaches maximum length at approximately $\phi=-10^{\circ}$. At this angle, the elbow joint angle is approximately $100^{\circ}$ which is the angle of the onset of the extension. The bottom left panel (c) shows the relationships between the stretch length and the angle $\phi$ when the passive components have the natural length at $\phi=0$. If the passive components had natural length at another angle, the curve of the relationship would shift vertically. The bottom right panel $(\mathrm{d})$ shows the relationships between the torque and the angle $\phi$. The solid line denotes the torque calculated from the moment arm shown in (b) and the stretch length shown in (c). The dotted line denotes the torque approximated by the parabola. The dotted lines fits with the solid line well. Even if the stretch length was shifted vertically, the torque would provide similar shape of curve, although it would be biased.

\subsection{IEMG and Elastic Coefficients The elas-}
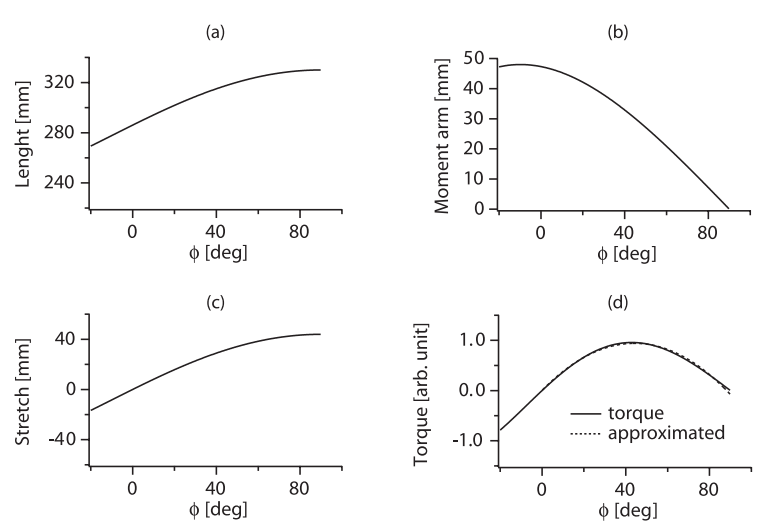

Fig. 8. Relationships among muscle length, moment arm, stretch length, torque and the angle $\phi$. (a) muscle length, (b) moment arm, (c) stretch length, (d) torque. The solid line representing torque is calculated from (b) and (c). The dotted line is the approximation of the solid line by the parabola. tic coefficients were not evaluated directly. For the healthy subject, it was impossible to separate the IEMGdependent elasticity $\left(a_{1}, a_{2}\right.$ and $\left.a_{3}\right)$ and the independent elasticity $\left(k_{0}\right.$ and $\left.k_{1}\right)$. Some of IEMGs of the healthy subjects had almost constant values, indicating that the IEMG-independent elasticity was distributed to both $k_{0}$ and IEMG-dependent elasticity with an unknown ratio. For the hemiplegic subjects, the IEMGs increased during forearm extension. The activated muscle differed among the subjects. It is generally accepted that the activation pattern of each muscles is related to those of other muscles, the elbow joint angle, angular velocity and so on. Therefore it was hard to assume the linear independence of the muscle activation.

\subsection{Time Constant of Normalized Elastic} Torque Considering a short time period, Eq. 7 is a linear second order equation because $k$ can be regarded as a constant value. One of the solution $\theta(t)$ is Eq. 11:

$$
\begin{gathered}
\theta(t)=z\left\{1-\frac{1}{\sqrt{1-\zeta^{2}}} e^{-\zeta \omega_{0} t} \sin \left(\sqrt{1-\zeta^{2}} \omega_{0} t+\psi\right)\right\} \\
\tan \psi=\frac{\sqrt{1-\zeta^{2}}}{\zeta} \ldots \ldots \ldots \ldots \ldots \cdots \cdots \cdot(11) \\
\omega_{0}=\sqrt{\frac{k}{I^{\prime}}}, \quad \zeta=\frac{\eta}{2 \sqrt{k I^{\prime}}}, \quad z=\frac{m g r}{k}
\end{gathered}
$$

The normalized elastic torque, $k \theta(t) / m g r(=\theta(t) / z)$, is equal to Eq. 10. The time constant $\tau$ is $1 / \zeta \omega_{0}$ $\left(=2 I^{\prime} / \eta\right)$. If $k$ were a constant value, it would be possible to evaluate $2 I^{\prime} / \eta$. However, $k$ depended on $\theta$ and IEMGs in this study. Therefore, we approximated the normalized elastic torque with the dumped sinusoid and evaluated the time constant of the approximated curve.

\section{Conclusion}

We have proposed a new technique for modeling the step-like response of elbow extension in the hemiplegic subjects. The response was approximated with a model whose elastic coefficients depend on the elbow joint angle and muscle activity. The normalized elastic torque was calculated and then it was approximated with a dumped sinusoid. The relationship between the inverse of the time constant and the natural frequency, the T-F plot, had some clusters depending on the modified Ashworth scales of the subjects. The results suggest that the T-F plot can be a quantitative index of spasticity.

(Manuscript received Dec. 20, 2004, revised June 16, 2005)

\section{References}

(1) I. Odéen and E. Knuttson: "Evaluation of the effects of muscle stretch and weight load in patients with spastic paraplegia", Scand. J. Rehab. Med., Vol.13, pp.117-121 (1981)

(2) G. C. Agarwal and G. L. Gottlieb: "Oscillation of the human ankle joint in response to applied sinusoidal torque on the foot", J. Physiol., Vol.268, pp.151-176 (1977)

(3) I. W. Hunter and R. E. Kearney: "Dynamics of human ankle stiffness: Validation with mean ankle torque", J. Biomech., Vol.15, pp.747-752 (1982)

(4) P. M. H. Rack, H. F. Ross, A. F. Thilmann, and D. K. Walters: 
"Reflex responses at the human ankle: The importance of tendon compliance", J. Physiol., Vol.344, pp.503-524 (1983)

( 5 ) J. F. Lehmann, R. Price, B. J. deLeteur, S. Hinderer, and C. Traynor: "Spasticity: Quantitative measurements as a basis for assessing effectiveness of therapeutic intervention", Arch. Phys. Med. Rehabil., Vol.70, pp.6-15 (1989)

(6) C. Sköld, K. Harms-Ringdahl, C. Hultling, R. Levi, and Å. Seiger: "Simultaneous Ashworth measurements and electromyographic recording in tetraplegic patients", Arch. Phys. Med. Rehabil., Vol 79, pp.959-965 (1998)

(7) F. Pisano, G. Miscio, C. D. Conte, D. Pianca, E. Candeloro, and R. Colombo: "Quantitative measures of spasticity in poststroke patients", Clin. Neurophysiol., Vol.111, pp.1015-1022 (2000)

(8) P. de Leva: "Adjustments to Zatsiorsky-Seluyanov's segment inertia parameters", J. Biomech., Vol.29, pp.1223-1230 (1996)

(9) P. de Leva: "Joint center longitudinal positions computed from a selected subset of Chandler's data", J. Biomech., Vol.29, pp.1231-1233 (1996)

(10) A. Faszczewski, K. Kȩdzior, and C. Rzymkowski: "Modeling of muscles co-operation under dynamic condition", presented at Second World Congress of Biomechanics, I-179a, Amsterdam (1994)

(11) A. G. Feldman: "Functional tuning of the nervous system with control of movement or maintenance of a steady posture -II. controllable parameters of the muscle", Biofizika, Vol.11, pp.565-578 (1966)

(12) Y. Gait, J. Mizrahi, W. S. Levine, and J. Chen: "Simulation of distal tendon transfer of the biceps brachii and the brachialis muscles", J. Biomechanics, Vol.27, pp.1005-1014 (1994)

(13) E. J. van Zuylen, A. van Velzen, and J. J. D. van der Gon: "A biomechanical model for flexion torques of human arm muscles as a function of elbow angle", J. Biomechanics, Vol.21, pp.183-190 (1988)

(14) T. Uchiyama, T. Bessho, and K. Akazawa: "Static torqueangle relation of human elbow joint estimated with artificial neural network technique", J. Biomechanics, Vol.31, pp.545554 (1998)

(15) K. Akazawa, K. Nire, and K. Fujii: "Data Compression of Myoelectric Signal-Estimation of Force and Its Long-Term Monitoring", Systems and Computers in Japan, Vol.20, No.4, pp.48-58 (1989)

(16) K. Akazawa, R. Okuno, and H. Kusumoto: "Relation between intrinsic viscoelasticity and activation level of human finger muscle during voluntary isometric contraction", Frontiers of Medical and Biological Engineering, Vol.9 , No.2, pp.123-135 (1999)

\section{Appendix}

\section{Calculation of Parameters}

Substituting Eq. 8 into Eq. 7 and then rewriting it with sampled data yields Eq. A1.

$$
\Omega \mathbf{p}=\mathbf{q}
$$

where

$\Omega=\left[\begin{array}{ccccccc}\ddot{\theta}_{1} & \dot{\theta}_{1} & \theta_{1} & \theta_{1}^{2} & \theta_{1} e_{1_{1}} & \theta_{1} e_{2_{1}} & \theta_{1} e_{3_{1}} \\ \ddot{\theta}_{2} & \dot{\theta}_{2} & \theta_{2} & \theta_{2}^{2} & \theta_{2} e_{1_{2}} & \theta_{2} e_{2_{2}} & \theta_{2} e_{3_{2}} \\ \vdots & \vdots & \vdots & \vdots & \vdots & \vdots & \vdots \\ \ddot{\theta}_{N} & \dot{\theta}_{N} & \theta_{N} & \theta_{N}^{2} & \theta_{N} e_{1_{N}} & \theta_{N} e_{2_{N}} & \theta_{N} e_{3_{N}}\end{array}\right]$,

$\mathbf{p}=\left[\begin{array}{c}I^{\prime} \\ \eta \\ k_{0} \\ k_{1} \\ a_{1} \\ a_{2} \\ a_{3}\end{array}\right], \quad \mathbf{q}=\left[\begin{array}{c}m g r \\ m g r \\ \vdots \\ m g r\end{array}\right]$

In Eq. $\mathrm{A} 1, \theta_{i}, e_{1_{i}}, e_{2_{i}}$ and $e_{3_{i}}(i=1,2, \cdots, N)$ are sampled $\theta(t), e_{1}(t), e_{2}(t)$ and $e_{3}(t)$, respectively. $N$ is the number of sampled data. The acceleration $\ddot{\theta}_{i}$ $(i=1,2, \cdots, N)$ and the velocity $\dot{\theta}_{i}(i=1,2, \cdots, N)$ were calculated from the observed elbow angle $\theta_{i}$ by the numerical derivation.

The sum of the squares of error shown in Eq. A2 shows the minimum when partial differentiation of $E$ with respect to the parameters $\left(\partial E / \partial I^{\prime}, \partial E / \partial \eta, \partial E / \partial k_{0}\right.$, $\partial E / \partial k_{1}, \partial E / \partial a_{1}, \partial E / \partial a_{2}$ and $\left.\partial E / \partial a_{3}\right)$ are zero.

$$
\begin{aligned}
E= & \sum_{i=1}^{N}\left(m g-I^{\prime} \ddot{\theta}_{i}-\eta \dot{\theta}_{i}-k_{0} \theta_{i}-k_{1} \theta_{i}^{2}\right. \\
& \left.-a_{1} e_{1_{i}} \theta_{i}-a_{2} e_{2_{i}} \theta_{i}-a_{3} e_{3_{i}} \theta_{i}\right)^{2} \ldots \ldots .
\end{aligned}
$$

The equations where the partial differentiation of $E$ with respect to the parameters are zero yield Eq. A3.

$$
\Omega^{T} \Omega \mathbf{p}=\Omega^{T} \mathbf{q}
$$

where $T$ denotes the transpose of a matrix.

The parameters are obtained by solving the simultaneous equation of Eq. A3.

Takanori Uchiyama (Member) was born on 29 of January,

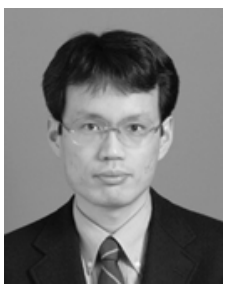
1965 and received the B. S. and M. S. degree in instrumentation engineering from Keio University, Japan, in 1987 and 1989, respectively. $\mathrm{He}$ received the $\mathrm{Ph}$. D degree in Biomedical Engineering from Keio University in 1992. From 1992 to 1997, He was a member of the faculty at Kobe University. Since 1997 he has been a member of the faculty at Keio University, where he is currently an associate professor at the department of applied physics and physico-informatics. His research interests include neuromuscular system, biomechanics and rehabilitation engineering. He is currently the member of IEEE, IEEJ, SICE and JSMBE.

Ryusei Uchida (Non-member) was born in 1957 and gradu-

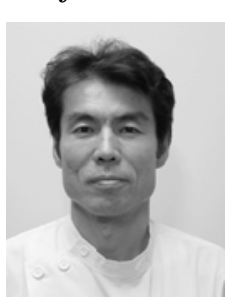
ated from University of Occupational and Environmental Health, Japan in 1984. He was a research fellow of Department of Anatomy, University of Occupational and Environmental Health in 1990. He worked as a medical Doctor of Department of Rehabilitation, KantoRosai Hospital from 1991 and as the Director of Department of Rehabilitation from 1995. His specialty is the rehabilitation of the patients with spinal cord injury and cerebral stroke. He is currently the member of IMSOP, JARM and JOA. 